\title{
Time-Frequency Enhancement Technique for Bevel Gear Fault Diagnosis
}

\author{
Dennis Hartono ${ }^{1}$, Dunant Halim ${ }^{1}$, Achmad Widodo ${ }^{2}$ and Gethin Wyn Roberts ${ }^{3}$ \\ ${ }^{1}$ Department of Mechanical, Materials and Manufacturing Engineering, The University of Nottingham Ningbo China, China 315100 \\ ${ }^{2}$ Department of Mechanical Engineering, Diponegoro University, Semarang, Indonesia 50275 \\ ${ }^{3}$ Department of Civil Engineering, The University of Nottingham Ningbo China, China 315100
}

\begin{abstract}
Gear failure is one of the most common causes of breakdown in rotating machineries. It is well known that vibration signals from machineries can be effectively used to detect certain gear faults. Yet it is still not an easy task to find a symptom that reflects a particular fault from vibration signals. This paper presents an advanced time-frequency signal processing technique for extracting important gear fault information from the vibration signal that is heavily corrupted by measurement noise. Experiments were performed on a bevel gearbox test rig using vibration measurements. The Time Synchronous Average (TSA) was initially utilized to eliminate all asynchronous component of vibration signal obtained from the gear. The Continuous Wavelet Transform (CWT) method was then used to capture the non-stationary behaviour of the impulse signal generated from the broken bevel gear tooth. It was shown that the diagnosis method using the Continuous Wavelet Transform combined with Time Synchronous Averaging outperformed the conventional spectral analysis, capable of identifying the angular location of broken teeth in the gear.
\end{abstract}

\section{Introduction}

Diagnostic methods using vibration from geared systems have provided a significant contribution in preventing major failures in rotating machineries. However, most signal processing algorithms that have been developed for vibration diagnostic process rely on the assumption that the vibration signal is stationary. The complexity of vibration signal, comprising both transient and stationary components, can lead to insufficiency of these diagnostic methods to accurately detect gearbox faults from vibration signal.

It is well known that the main component in gearbox vibration signals is the gearmesh frequency, its harmonics and their sidebands around the gearmesh frequency. The modulation sidebands that are present around the fundamental frequency or harmonics of the tooth meshing frequency can provide useful information of gear defect locations [1]. Moreover, the amplitude of these sidebands can offer a quantitative measure of the severity of the gear fault [2]. However when the defects need to be identified in term of their angular locations most conventional methods, such as the Fast Fourier Transform (FFT) and Cepstrum, are not capable to provide such information accurately. Moreover, FFT assumes the underlying signal is a stationary signal, whereas the impulse generated by the gear fault is typically a transient signal. In this case, the use of timefrequency analysis has been proved to be able to detect the transient phenomena [3, 4], e.g. the impulse response generated from faulty gear. There is the need of using more sophisticated signal processing methods to provide such useful diagnostics information.

The objective of this work is to compare several advanced time-frequency techniques such as Short Time Fourier Transform and Continuous Wavelet Transform to capture the transient vibration signals generated by the gear fault accurately. Initially, the Time Synchronous Averaging technique was utilized to extract the gear fault feature from the vibrations signals that were heavily corrupted by measurement noise. The Fast Fourier Transform was utilized to extract the sidebands around the gearmesh frequencies that indicated which particular shaft the faulty gear was attached. Finally, STFT and CWT methods were compared to offer an accurate identification of the faulty gear angular.

\section{Theoretical background}

\subsection{The time synchronous averaging}

The Time Synchronous Averaging is the process of ensemble averaging by sampling of the vibration signal according to the rotation of a particular gear of interest. In practice it will contain only components which are synchronous with the revolution of the gear in question. This method requires a rotational reference signal to determine angular position of the required gear. The meshing vibration $\mathrm{x}(t)$ is determined by the signal 
average of one of the gears with $T$ teeth, which contains the fundamental frequency and its harmonics associated with the tooth meshing frequency $\left(T * f_{s}\right)$ as formulated below:

$$
\mathrm{x}(t)=\sum_{m=0}^{M} X_{m} \cos \left(2 \pi m T f_{s} t+\emptyset_{m}\right)
$$

where $X_{m}$ is the amplitude at the $m$-th harmonic frequency, $f_{\mathrm{s}}$ is the rotational frequency of the gear and $\emptyset_{m}$ is the initial phase. When a local defect occur in a gear, the changes in vibration signal will be observed because of the stiffness between neighbouring teeth will be influenced by the fault $[5,6]$. As a result the amplitude and phase modulation will occur in the meshing vibration $\mathrm{x}(t)$ as [5-8]:

$$
\mathrm{g}(t)=\sum_{m=0}^{M} X_{m}\left(1+a_{m}(t)\right) \cos \left(2 \pi m f_{s} t+\emptyset_{m}+b_{m}(t)\right)
$$

where

$$
\begin{aligned}
& a_{m}(t)=\sum_{n=0}^{N} A_{m n} \cos \left(2 \pi n f_{s} t+\alpha_{m n}\right) \\
& b_{m}(t)=\sum_{n=0}^{N} B_{m n} \cos \left(2 \pi n f_{s} t+\beta_{m n}\right)
\end{aligned}
$$

here, $a_{m}$ and $b_{m}$ are the amplitude and phase modulation functions caused by the changes in vibration signals due to the defect. $\alpha_{m n}$ and $\beta_{m n}$ are the phase angle of amplitude and phase modulation functions respectively. $A_{m n}$ and $B_{m n}$ are the amplitude of each harmonic in the amplitude and phase modulation function respectively. The index $m$ indicates that the modulation function may differ within each harmonic $m$. The index $n$ represents the harmonic index of the amplitude and phase modulation functions with respect to gear shaft rotation frequency.

\subsection{The Short Time Fourier Transform (STFT)}

The Short Time Fourier Transform is achieved by breaking down the signal into several overlapping usual Fourier Transform analysis. It is assumed that the underlying segmented signal will behave as a stationary signal so that the FFT assumption can be validated in this particular segment. STFT is formulated as $[3,9]$

$$
\mathrm{X}(t, f)=\int_{-\infty}^{\infty} \mathrm{x}(\tau) \mathrm{w}(t-\tau) e^{-j \pi f \tau} d \tau
$$

where $\mathrm{w}(t-\tau)$ is the window function and $\tau$ is the variable that slides the window across the underlying waveform.

\subsection{The Continuous Wavelet Transform (CWT)}

The continuous wavelet transform $W T_{x}(t, s)$ of a signal $\mathrm{x}(t)$ is defined as the convolution integral of $\mathrm{x}(t)$ with $\mathrm{a}$ translated and dilated version of mother wavelet function $\psi(t)$,

$$
W T_{x}(t, s)=\int_{-\infty}^{\infty} \mathrm{x}(\tau) \sqrt{s} \psi(s(t-\tau)) \mathrm{d} \tau
$$

where $s$ and $t$ are the scale and time parameters that produce the respective dilation and translation of the mother wavelet function [10]. One of the important considerations in CWT is the proper choice of the mother wavelet function that is determined by the characteristics of the underlying signals. It is well known that the morlet wavelet is suitable for gearbox fault diagnosis $[2,4,11-$ 13] because it has a similiar shape to that of the impulse signal. There are several types of morlet wavelet used in the existing literatures however, and the morlet wavelet used in this paper is formulated as [14]:

$$
\psi(x)=\frac{1}{\sqrt{\pi F b}} e^{2 i \pi F_{c} x} e^{\frac{-x^{2}}{F b}}
$$

where $F_{b}$ is the positive bandwith parameter and $F_{c}$ is the central frequency of the morlet wavelet.

\section{Gearbox experimental setup}

Vibration measurements from two pairs of a bevel gear unit from a gearbox test rig were performed. One of the pairs was with a broken tooth and another was without it. The gearbox test-rig from the Machinery Fault SimulatorSpectra Quest located at Diponegoro University was used to conduct the experiment. A magnetic brake was incorporated as a load. Three accelerometers sensor were used to capture the vibration signals from the test rig, although only the axial vibration measurement will be analysed in this work. The picture of the gearbox test-rig and accelerometers attached on it are shown in Fig. 1.

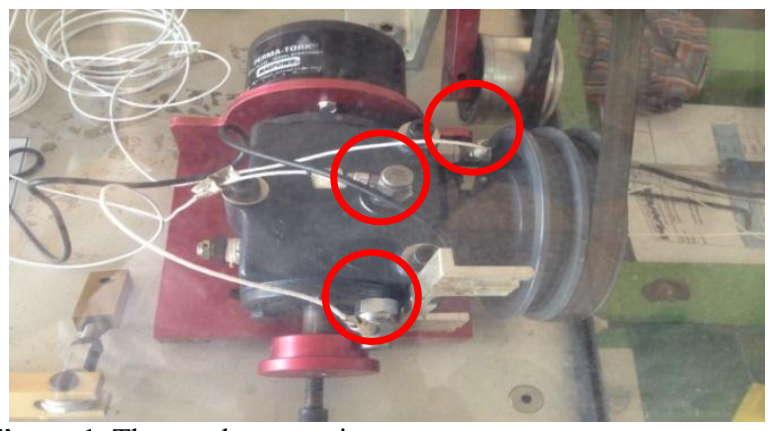

Figure 1. The gearbox test rig.

In this work the tooth breakage was introduced and the results from the faulty gear were compared with the healthy gear. The figure of the broken gear that is used in this work is shown in Fig. 2.

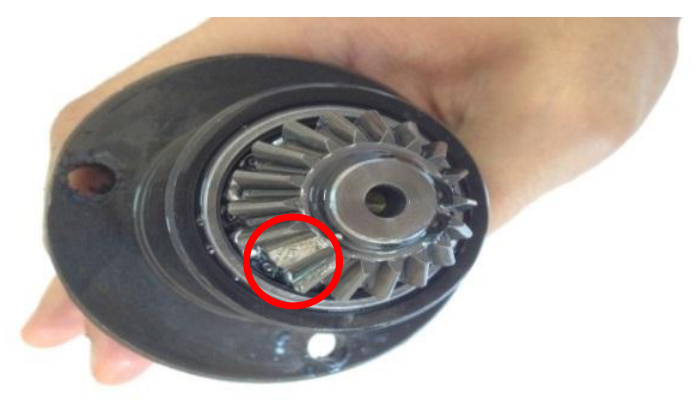

Figure 2. The gear with faulty tooth.

The sampling rate used to sample the vibration signals was $5.12 \mathrm{kHz}$. Eight seconds length data was gathered for each measurement performed in this experiment. Moreover, a tachometer sensor from Lattice Instruments 
was also used to provide the phase information from the gear. This information will be used for pre-processing the vibrations signal by TSA method to eliminate the asynchronous component from the faulty gear under consideration.

\section{Results and discussions}

\subsection{Vibration measurements from the test rig}

There were two sets of vibrations signal acquired from the gearbox test rig. The vibration signals (in terms of axial acceleration output from the accelerometer) from the healthy gearbox and the faulty gearbox are presented in Figs. 3 and 4, respectively.

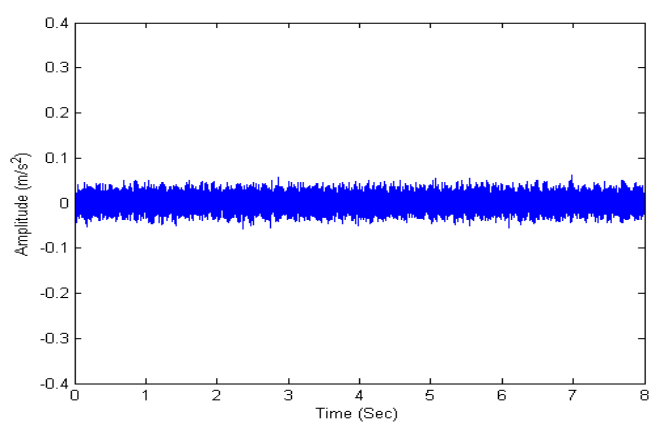

Figure 3. Vibration signal from a healthy gearbox.

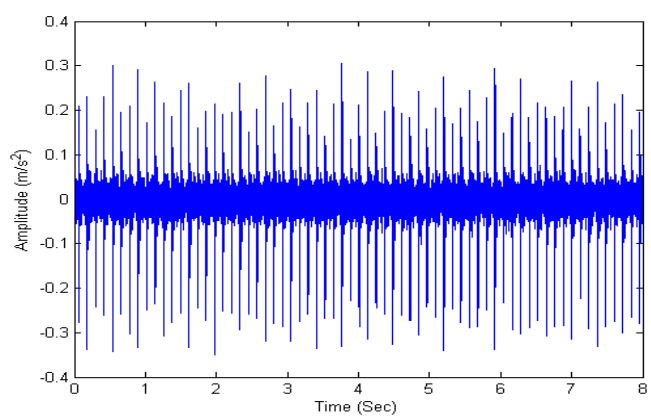

Figure 4. Vibration signal from a faulty gearbox.

As can be clearly seen from Figs. 3 and 4, there are a slight increase in the amplitude of the vibration signal from the broken gear. An impulse-like response (spikes) can occur when the faulty gear tooth makes a contact with the other gear [1]. However, it was difficult to identify the periodicity of this particular spike, which can correspond to the faulty gear tooth [15], solely by a direct visual inspection of the plot. Moreover this information is not enough to provide location the defect gears in terms of its angular location in the gear. Instead of the change in amplitude of the vibration signal, there is no further useful information can be gathered from these two plots.

\subsection{Pre-Processing by TSA}

Based on the obtained vibration, TSA can then be utilized to extract the periodic component that gets buried in the noise and eliminate all nonsynchronous vibration components. It represents an estimate of the meshing vibration over one revolution of a gear of interest. The
TSA signal of a healthy gear is presented in Fig. 5, which clearly shows a regular amplitude pattern. In general, the TSA of a healthy gear is usually composed of gear meshing waveforms modulated by some low shaft order (first or second) functions, where the shaft order is an integer multiple of the rotational speed of the shaft.

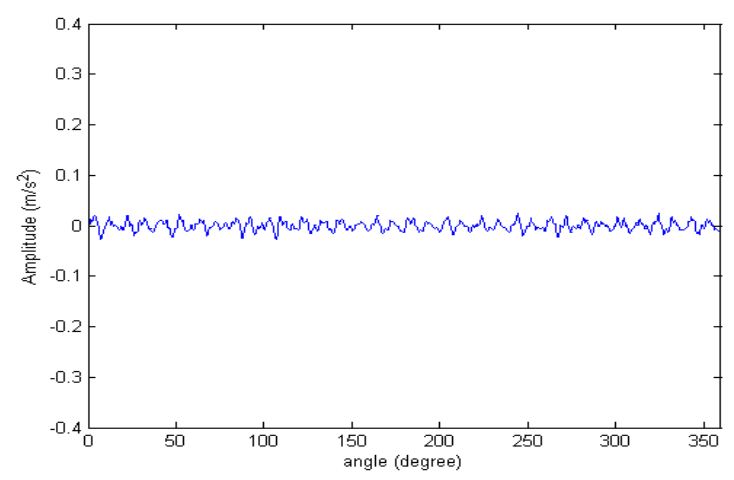

Figure 5. TSA vibration signal from a healthy gearbox.

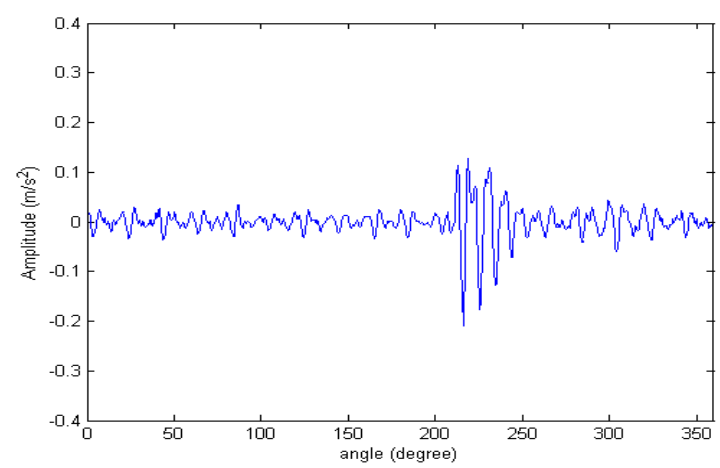

Figure 6. TSA vibration signal from a faulty gearbox.

On the other hand, the TSA of the faulty gear reveals a significant change in vibration amplitude at rotational angle of around $220^{\circ}$, caused by a tooth breakage as shown in Fig. 6. This impulse-like signal was generated by the faulty tooth when it meshed with other gears, which was observable in the TSA signal. These results demonstrate the ability of TSA to offer a precise location of the faulty tooth, which is not observable in the raw vibration signal.

Another way of interpretation of the vibration signal is in frequency domain by the use of FFT. FFT plot offers diagnostic information of the health of gearbox, particularly indicated through the sidebands that appear around the gearmesh frequency and its harmonics. For healthy gears, the FFT plot of the TSA signal is only composed of the fundamental gearmesh frequency and the low shaft order peaks (1-10 orders). When the fault occurs, the FFT of TSA signal will produce higher order of sidebands. As previously mentioned in the introduction, when a fault occurs, the amplitude of the sidebands increases and its spacing from the corresponding gearmesh harmonics corresponds to the location of the fault. Figs. 7 and 8 showed the FFT plots of the healthy gear and broken gear respectively. It is obvious that when the fault occurs there are more high amplitude sidebands that appear around second, third and fourth gearmesh frequencies compared to the normal FFT of 
healthy gear. However, several spurious peaks can still be observed appearing in FFT plot. This is due to spectral leakage and smearing as inherent drawbacks of analyzing non-stationary signal with FFT. It is happened because when the fault occurs, the speed and load applied to the gearbox system are not constant anymore at that particular instant of time, so the stationary assumption that FFT relies on does not hold anymore.

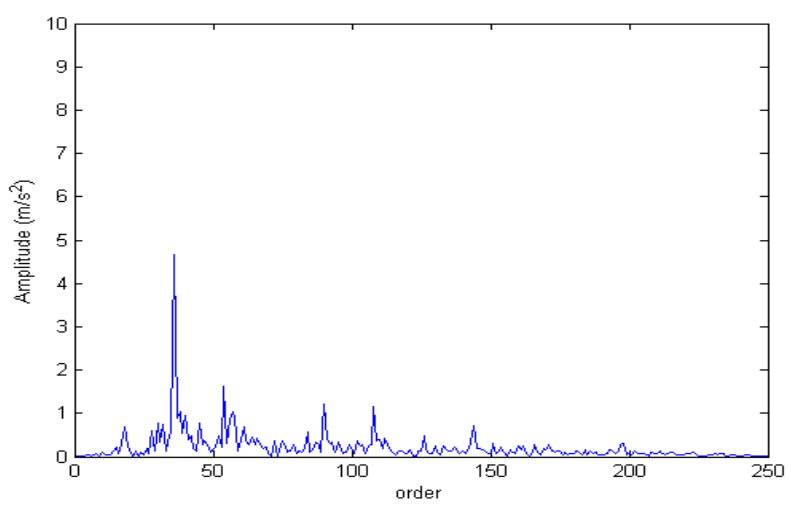

Figure 7. FFT of TSA vibration signal from a healthy gearbox.

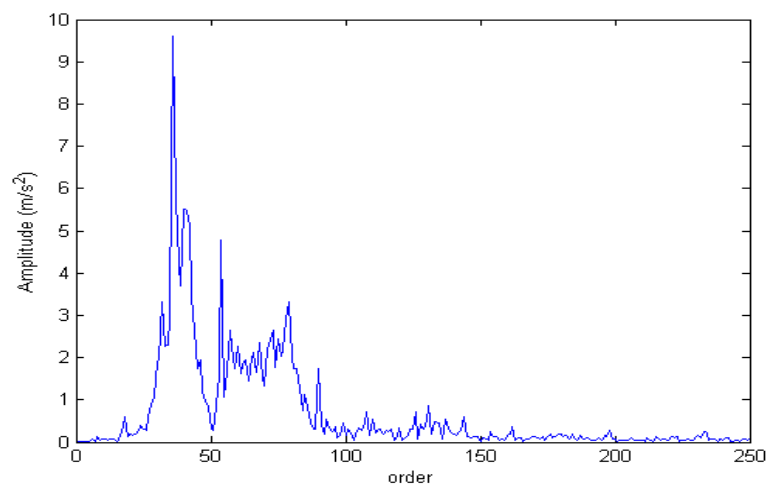

Figure 8. FFT of TSA axial vibration signal from a faulty gear.

\subsection{Time-Frequency Method}

From the drawback of the FFT, this work proposes a time-frequency technique to analyse the transient phenomena generated by the impulse signal in TSA. STFT plots of the healthy and broken gear are respectively shown in Fig. 9 and 10.

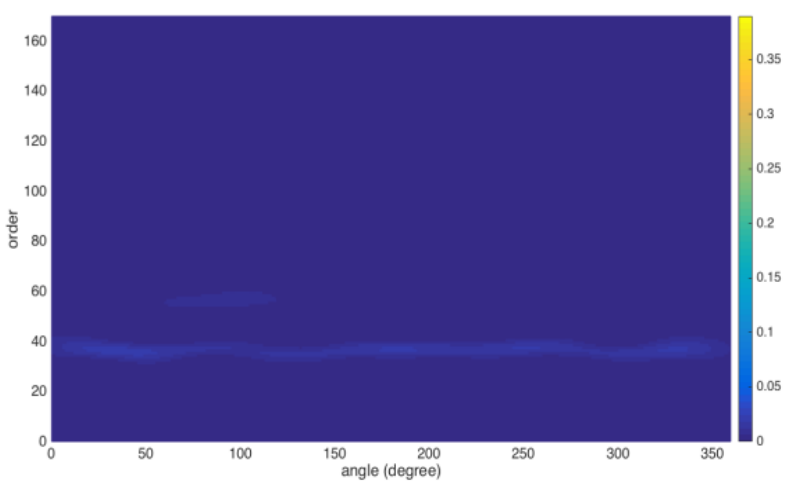

Figure 9. STFT of TSA vibration signal for a healthy gearbox.

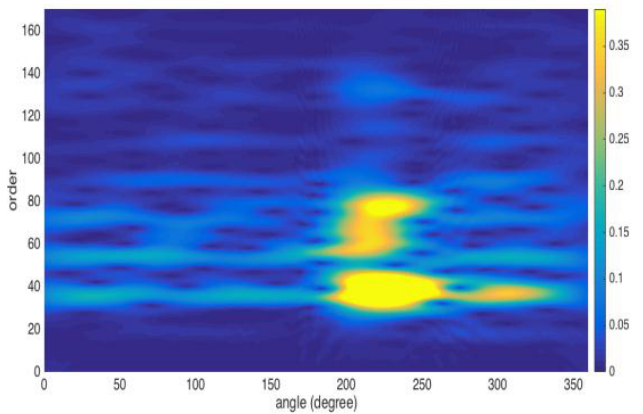

Figure 10. STFT of TSA vibration signal for a faulty gearbox.

Fig. 10 clearly indicated that STFT was able to capture the transient phenomena of the impulse signal when the faulty tooth was meshed with its neighbour. This is not possible to achieve using the usual Fourier Transform because the angle information is lost. Moreover, it also can distinguish between the healthy and broken gear TSA signals by showing the higher amplitude intensity observed from the STFT plot for the broken gear, compared to STFT plot for the healthy gear. The appearance of more sidebands and $4^{\text {th }}$ harmonics $\left(72^{\text {nd }}\right.$ order) of the gearmesh frequency that do not appear in the results of the healthy gearbox can be seen from Fig. 10. STFT plot, however still provides an absurd interpretation by showing a significant intensity at an angle larger than $250^{\circ}$ which does not correspond to any fault in the gear.

To address the drawback of STFT, the continuous wavelet transform of the TSA signal was investigated. Figs. 11 and 12 show the CWT plot of TSA vibration signal from the healthy and faulty gearboxes, respectively.

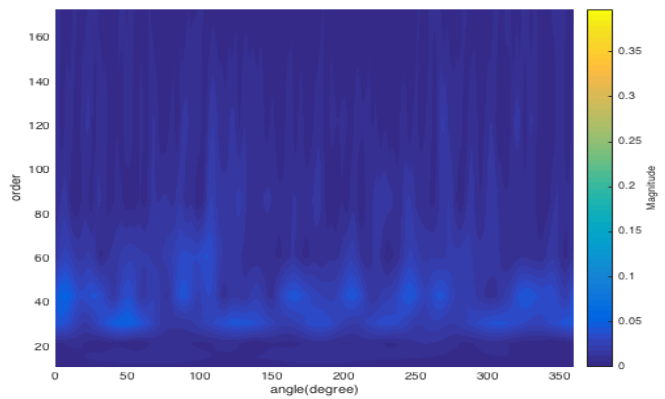

Figure 11. CWT of TSA axial vibration signal from a faulty gearbox.

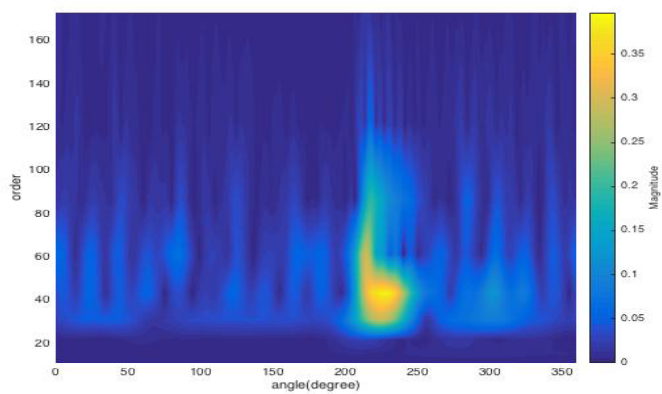

Figure 12. CWT of TSA vibration signal from a faulty gearbox.

It can clearly be seen that the contrast of the intensity between the vibration signals from the healthy and faulty gearboxes from Figs. 11 and 12. It is demonstrated that 
CWT not only able to distinguish the normal gear and broken gear vibration signals but also offer a better time resolution compared to STFT. CWT can better indicate that the fault occurs at the angle between $200^{\circ}$ and $250^{\circ}$. It is thus shown that the joint angle-frequency representation is quite beneficial to identify the location of the gear fault, in terms of angular location. Such information cannot be obtained using the conventional spectral analysis like FFT, which can only offer the frequency content of the signal at the expense of the time information.

\section{Conclusions}

This study has demonstrated the usefulness of timefrequency signal processing of vibration signal for diagnosing the gearbox health based on experimental results from a bevel gearbox test rig. It has been shown that the TSA method had the ability to identify the angular location of the gear fault, which was not possible to obtain by using the raw vibration signal alone. FFT was used to provide a different representation of vibration signal in frequency domain. It was found that the information about angular location of gear fault was lost once the FFT analysis was performed. Moreover, FFT analysis was not a suitable method to analyse transient behaviour generated by broken tooth. In this work, STFT and CWT were utilized to provide a better interpretation of the impulse signal obtained from the TSA method in the joint time frequency domain. Although STFT of TSA signals were able to distinguish the difference between the normal and faulty gearboxes, it was still not good enough to offer an accurate angular location of the faulty gear tooth due to the need to choose the appropriate parameter for the window function. On the other hand, CWT was not only able to distinguish TSA signals from healthy and faulty gearboxes, but can also provide a better time resolution of the TSA vibration signal compared to STFT. The experimental results in this work have demonstrated that the combination of TSA and CWT is a reliable technique for enhancing gearbox fault diagnosis by offering an accurate angular location of the gear fault.

\section{Acknowledgment}

The authors gratefully acknowledge the support provided by Ningbo Science and Technology Bureau-International Cooperation Programme (Project: 2012D10029), China.

\section{References}

1. R. B. Randall, J. Mech. Des 104, 259-267 (1982)

2. G. Dalpiaz, A. Rivola, R. Rubini, Mech. Sys. and Sig. Proc, 84, 387-412 (2000)

3. W. J. Wang, P. D. McFadden, Mech. Sys and Sig. Proc, 7, 193-203 (1993)

4. W. J. Staszewski, G. R. Tomlinson, Mech. Sys and Sig. Proc, 8, 289-307 (1994)

5. P. D. McFadden, J. Vib. Aco. Strss. Real. 108, 165170 (1986)

6. P. D. McFadden, Mech. Sys and Sig. Proc, 2, 403409 (1988)

7. R. B. Randall, J. Mech, Des 104, 259-267 (1982)

8. P. D. McFadden, J. D. Smith, Proocedings of the Institution of Mechanical Engineers 199, 287-292 (1985)

9. L. Cohen, Proceedings of the IEEE 77, 941-981 (1989)

10. W. J. Wang, P.D. McFadden, J. Snd. Vib 192, $927-$ 939 (1996)

11. D. Bouhlabal, M. F. Golnaraghi, F. Ismail, Mech. Sys and Sig. Proc, 13, 423-436 (1999)

12. W. Q. Wang, F. Ismail, M. F. Golnaraghi, Mech. Sys. Sig. Proc, 15, 905-922 (2001)

13. H. Zheng, Z. Li, X. Chen, Mech. Sys. Sig. Proc, 16, 447-457 (2002)

14. A. Teolis, Computational Signal Processing with Wavelets. 65 (1998)

15. C. K. Sung, H. M. Tai, C. W. Chen, Mech. Mach. Thry 35, 1169-1182 (2000) 